\title{
A Preisach Model for Monotonic Tension Response of Structural Mild Steel with Damage
}

\author{
Petar Kneževići ${ }^{\star}$, Dragoslav Šumarac², Zoran Perović², Ćemal Dolićanin'1, Zijah Burzić3 \\ ${ }^{1}$ Department of Technical Science, Faculty of Civil Engineering, State University of Novi Pazar, Vuka Karadžića bb, 36300 Novi \\ Pazar, Serbia \\ 2 Department of engineering mechanics and theory of structures, Faculty of Civil Engineering, University of Belgrade, Bulevar \\ Kralja Aleksandra 73, 11120 Belgrade, Serbia \\ ${ }^{3}$ Military Technical Institute, Ratka Resanovića 1, 11000 Belgrade, Serbia \\ * Corresponding author, e-mail: petar.knezevic.dunp@mail.com
}

Received: 07 August 2019, Accepted: 23 January 2020, Published online: 18 February 2020

\begin{abstract}
This paper presents the new type of Preisach model that describes the elastoplastic behavior of structural mild steel under axial monotonic tension load with damage. Newly developed model takes into account elastic region, horizontal yield plateau, plastic hardening region, and softening region due to material damage under tension. In order to study the monotonic behavior of structural mild steel and find suitable material properties for the model, monotonic axial tensile tests up to the failure are carried out. Tests are conducted on specimens of the three most common types of European structural steel S235, S275, and S355. The basis of the model represents a mathematical description of material single crystal monotonic axial behavior. In the multilinear mechanical model, a drop in stress, after achieving ultimate stress under tension is achieved by a negative stiffness element. The good agreement with experimental results is accomplished by parallel connection of infinitely many single crystal elements, forming the polycrystalline model. The model represents a good solution for common engineering practice due to its geometrical representation in form of Preisach triangle.
\end{abstract}

Keywords

monotonic axial strain, yield plateau, Preisach model, negative stiffness, damage

\section{Introduction}

Depending on the shape of the material stress-strain diagram, the behavior of the material can be classified into several groups. Apart from brittle materials, in which there is almost no elastic response, a large number of materials have a considerable elastic region in which the relationship between stress and strain is proportional to the elasticity modulus $E$, which is a material constant. The linear relation $\sigma-\varepsilon$ of the diagram is valid until the stress reaches the flow limit $\sigma_{T}$. Depending on their behavior after reaching the flow limit, elastic materials can be classified as ideal elastoplastic material and ideally elastoplastic material with hardening. Most type of steel can be classified in these two material groups. However, the behavior of low carbon steel, mild steel, some alloys of steel (Al-Mg), and some non-ferrous metals is characterized by an unstable elasto-plastic transition that deteriorates the formability and ductility of such materials. This phenomenon is the result of the separation of free atoms (usually carbon or nickel) and their pining at the existing and newly formed dislocations within the iron atoms matrix [1].

The behavior of these materials represents a combination of ideally elastoplastic material without hardening and ideally elastoplastic behavior with hardening and they are classified as Type 4 behavior [2]. The initial part of the stress-strain diagram is linear and proportional to the elasticity modulus $E$, up to reaching the stress value, called the upper yield point $\sigma_{u y}$, which corresponds to the unpinning of pinned dislocations in a crystal lattice. Achieving the $\sigma_{u y}$ is followed by a sudden drop in stress to the lower yield point $\sigma_{l y}$, after which local deformation band is formed. The formation of a horizontal plateau, an approximate size of $1 \%-3 \%$ of the total strain, represents a material instability from elastic to plastic deformations, called Lüders band phenomenon. 
Type 4 behavior of structural mild steel is caused by their microstructure and atomic lattice. Free carbon and nitrogen atoms surrounding dislocation are causing a high level of stress required to initiate dislocation propagation inside the crystal lattice. After the initiation of dislocation, its movement is relatively easy to proceed, forming a yield plateau, until a new realignment of the atoms within the crystal lattice occurs.

The upper and lower yield points, as well as the length of Lüders band (Lüders strain), depend on a large number of parameters, such as the size of the steel grain [3], the strain rate and the carbon content [4] Increasing the loading and strain rate increases the length of the plateau.

The value of the upper yield point $\sigma_{u y}$, is very sensitive to minor stress concentrations, alignment of the sample inside the jaw, and other test parameters, causing significant dispersal of test results. For that reason, the upper yield point is ignored, and the value of the lower yield point $\sigma_{l y}$ is taken for the stress at which the transition from elastic to the plastic region occurs.

Stress-strain response of structural mild steel up to ultimate stress $\sigma_{u}$ is characterized by a positive stiffness, where an increase in strain results in a corresponding increase in stress. After reaching maximum stress value $\sigma_{u}$ under tension, softening phenomena occurs, causing sample necking due to damage propagation. The post-peak softening stage exhibits negative stiffness. There are many examples of systems with a negative stiffness. However, the definition of these phenomena, by using elements with negative stiffness, is contemporary approach. Up to now, phenomena is modeled with system of springs [5], inclined rods [6], the effect of pre-buckled columns [7], the magnet [8], and the electromagnet [9]. The analysis of the systems with elements of negative stiffness is most often carried out using the standard methods with the change of the stiffness sign of the observed element [10].

In this paper, for modeling the axial response of monotonic loaded structural mild steel, Preisach model is used. Originally developed for defining hysteretic phenomena in magnetism [11], the model quickly found application in other fields of physics. The first implementation of this model in continuum mechanics describes the behavior of ductile materials under cyclic loading [12, 13]. Generally, the Preisach model is hysteretic operator used for defining cyclic behavior of ductile materials. It is pure mathematical operator [14], which maps input function $u(t)$ into output function $f(t)$ by:

$$
f(t)=\hat{\Gamma} u(t)=\iint_{\alpha^{3} \beta} G_{\alpha, \beta} u(t) \mu(\alpha, \beta) d \alpha d \beta,
$$

where $G_{\alpha, \beta}$ is an elementary hysteresis operator given in Fig. 1. Parameters $\alpha$ and $\beta$ are up and down switching values of the input, while $\mu(\alpha, \beta)$ is Preisach (weight) function.

In addition to the primary characteristic of a model to describe the cyclic behavior of materials, it is able to accurately describe the monotonic behavior of the material. Due to the complex behavior of the structural mild steel under cyclic loads, which is the result of the cyclic hardening and the Lüders band phenomenon, the monotonic and cyclic behavior of the considered steel must be observed separately. Existing models, except [15] are unable to define the effect of softening in a material that occurs as a result of tensile damage.

The Preisach model requires a uniquely defined relationship between the input and the output data, and due to the existence of a horizontal yield plateau, the deformation is imposed as the input of a newly defined model.

Existing models, mapping strain $\varepsilon(t)$ as input into stress $\sigma(t)$ as output, are based on bilinear working diagrams (Fig. 2). They are used for modeling cyclic and monotonic behavior of ideally elastoplastic materials and ideally elastoplastic materials with linear hardening and represent the basis for a new model.

The first part of the paper represents an introduction to the analyzed problem. In the Section 2 of this paper, basic expressions and considerations of the single crystal Preisach model under monotonic axial load are given. In the Section 3, the polycrystalline model is introduced and finally, model verification is shown in Section 4. The application of the proposed model is verified by comparison with experimental data obtained by testing cylindrical samples made of three types commonly used European structural steel S235, S275, and S355.

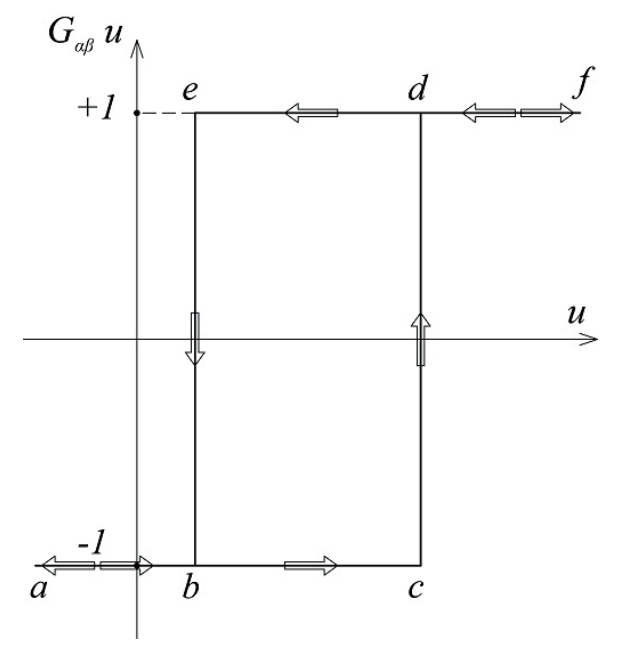

Fig. 1 Elementary hysteresis operator 
a)
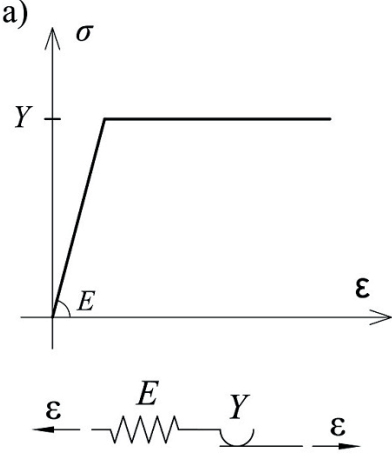

b)

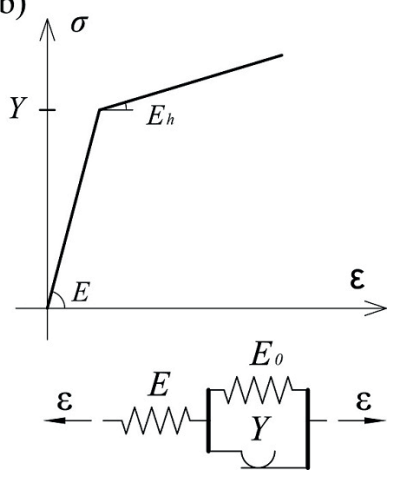

Fig. 2 Working diagrams and mechanical models for: a) ideally elastoplastic material; b) ideally elastoplastic material with hardening

2 Single crystal Preisach model and monotonic axial response of structural mild steel with damage

The behavior of structural mild steel under monotone and cyclic load are quite different. Monotonic loading is characterized by horizontal yield plateau which is vanishing under cyclic loading, due to phenomena of cyclic hardening. It must be noticed that structural mild steel behavior is different under compression and tension. Only under tension, damage occurs, causing the formation of the softening region after reaching ultimate stress $\sigma_{u}$.

A new mechanical model with the mentioned properties is presented in Fig. 3. Model is compiled of four Hook's spring elements, three Saint-Venant's slip elements, and two delay elements.

The first delay element allows the hardening delay by providing an empty shift of $\pm_{\varepsilon_{L}}$, after reaching yield stress $Y_{1}$. Last spring element has negative stiffness $h_{3}$, causing a drop in stress after reaching maximum stress $\sigma_{u}$ under tension. This phenomenon is allowed by the last delay element which is limited only in a region of tension, forming second yield plateau with length $\varepsilon_{d}$.

The appropriate stress-strain diagram displayed in Fig. 4 presents different behavior of single crystal model under compression and tension, where the softening region due to damage befalls only under tension.

The material properties of the mechanical model achieved by a parallel or regular connection of the spring elements are defined by Eq. (2).

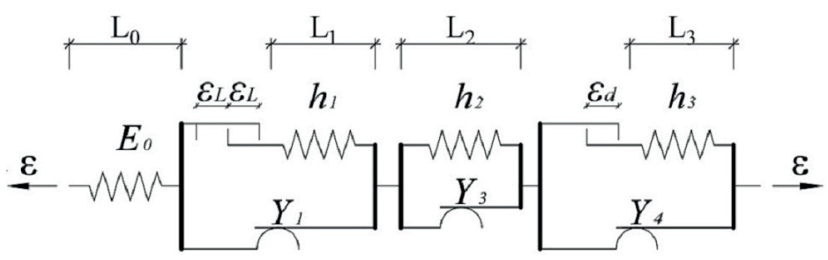

Fig. 3 Mechanical single crystal model

$$
\begin{aligned}
& E=E_{0}\left(L_{0}+L_{1}+L_{2}\right) / L_{0} \\
& E_{a}=E_{0} \cdot E_{1} /\left(E_{0}+E_{1}\right) \\
& E_{1}=h_{1}\left(L_{0}+L_{1}+L_{2}\right) / L_{1} \\
& E_{h}=E_{0} \cdot E_{1} \cdot E_{2} /\left(E_{0}+E_{1}+E_{2}\right) \\
& E_{2}=h_{2}\left(L_{0}+L_{1}+L_{2}\right) / L_{2} \\
& E_{D}=E_{0} \cdot E_{1} \cdot E_{2} \cdot E_{3} /\left(E_{0}+E_{1}+E_{2}+E_{3}\right) \\
& E_{3}=h_{3}\left(L_{0}+L_{1}+L_{2}\right) / L_{3}
\end{aligned}
$$

The stress-strain diagram for the single crystal material model shown in Fig. 3 and material characteristics, given by Eq. (2) is represented in Fig. 4, showing different behavior of structural mild steel under tension and compression monotonic axial load.

From the monotonic stress-strain curve, output function $f(t)$ is defined by Eq. (3):

$f_{\alpha, \beta}=f_{\alpha}-E(\alpha-\beta)$ for $\alpha-2 \varepsilon_{1}<\beta \leq \alpha$

$f_{\alpha, \beta}=f_{\alpha}-2 Y_{1}$ for $\alpha-2 \varepsilon_{2}<\beta \leq \alpha-2 \varepsilon_{1}$

$f_{\alpha, \beta}=f_{\alpha}-2 Y_{1}-E_{a}\left(\alpha-\beta-2 \varepsilon_{2}\right)$

for $\alpha-2 \varepsilon_{3} \leq \beta \leq \alpha$

$f_{\alpha, \beta}=f_{\alpha}-2 Y_{1}-2 E_{a} \varepsilon_{a}-E_{h} \times\left(\alpha-\beta-2 \varepsilon_{3}\right)$

for $\alpha-2 \varepsilon_{4} \leq \beta \leq \alpha-2 \varepsilon$

$f_{\alpha, \beta}=f_{\alpha}-2 Y_{4}$ for $-\varepsilon_{d} \leq \beta \leq \alpha-2 \varepsilon_{4}$

$f_{\alpha}+E_{D}\left(\alpha-2 \varepsilon_{5}\right)$ for $-\varepsilon_{\mathrm{d}} \leq \beta \leq \alpha-2 \varepsilon_{5} \wedge \alpha+\beta \geq 0$

where:

$$
\begin{aligned}
& \varepsilon_{1}=Y_{1} / E \\
& \varepsilon_{2}=Y_{1} / E+\varepsilon_{L} \\
& \varepsilon_{3}=Y_{1} / E+\left(\varepsilon_{L}+\varepsilon_{a}\right) \\
& \varepsilon_{4}=Y_{1} / E+\left(\varepsilon_{L}+\varepsilon_{a}+\varepsilon_{h}\right) \\
& \varepsilon_{5}=Y_{1} / E+\left(\varepsilon_{L}+\varepsilon_{a}+\varepsilon_{h}+\varepsilon_{d}\right)
\end{aligned}
$$

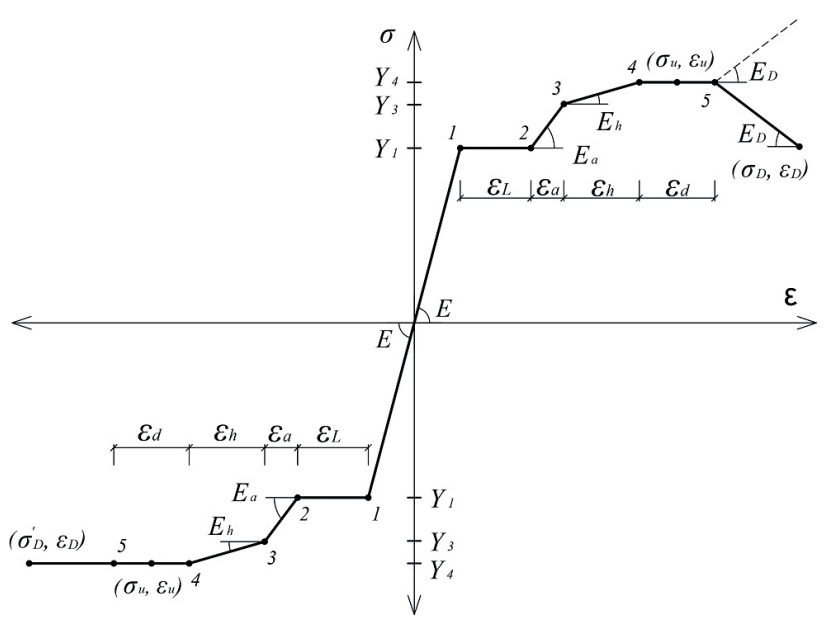

Fig. 4 The stress-strain diagram of structural mild steel single crystal under tension and compression 
According to [14], the Preisach function as the tangent slope of the first order transitional curve $f_{\alpha, \beta}$, is determined:

$$
\begin{aligned}
& \mu(\alpha, \beta)=\frac{1}{2} \frac{\partial^{2} f_{\alpha, \beta}}{\partial \alpha \partial \beta} \\
& \frac{E}{2} \cdot\left[\delta(\alpha-\beta)-\delta\left(\alpha-\beta-2 \varepsilon_{1}\right)\right]+\frac{E_{a}}{2} \cdot \delta\left(\alpha-\beta-2 \varepsilon_{2}\right) \\
& +\frac{E_{h}-E_{a}}{2} \delta\left(\alpha-\beta-2 \varepsilon_{3}\right)-\frac{E_{h}}{2} \cdot \delta\left(\alpha-\beta-2 \varepsilon_{4}\right) \\
& +\frac{E_{D}}{2} \delta\left(\alpha-\beta-2 \varepsilon_{5}\right) \cdot H(\alpha+\beta),
\end{aligned}
$$

where $H$ stands for Heaviside step function and $\delta$ for Dirac delta function.

This Preisach function is defined only along the lines $\alpha-\beta=0, \alpha-\beta-2_{\varepsilon 1}=0, \alpha-\beta-2_{\varepsilon 2}=0, \alpha-\beta-2_{\varepsilon 3}=0$, and $\alpha-\beta-2_{\varepsilon 4}=0$.

The input under tension is denoted by $\alpha$ and under compression as $\beta$.

Including the Preisach function $\mu(\alpha, \beta)$, defined by the Eq. (5), into the Eq. (1), the expression for the stress $\sigma(t)$, as the output $f(t)$, due to the deformation $\varepsilon(t)$ as the input $u(t)$, is defined:

$$
\begin{aligned}
& \sigma(t)=\frac{E}{2} \iint_{\alpha \geq \beta} \delta(\alpha-\beta) G_{\alpha, \beta} \varepsilon(t) d \alpha d \beta \\
& -\frac{E}{2} \iint_{\alpha \geq \beta} \delta\left(\alpha-\beta-2 \varepsilon_{1}\right) G_{\alpha, \beta} \varepsilon(t) d \alpha d \beta \\
& +\frac{E_{a}}{2} \iint_{\alpha \geq \beta} \delta\left(\alpha-\beta-2 \varepsilon_{2}\right) G_{\alpha, \beta} \varepsilon(t) d \alpha d \beta \\
& +\frac{E_{h}-E_{a}}{2} \iint_{\alpha \geq \beta} \delta\left(\alpha-\beta-2 \varepsilon_{3}\right) G_{\alpha, \beta} \varepsilon(t) d \alpha d \beta \\
& -\frac{E_{h}}{2} \iint_{\alpha \geq \beta} \delta\left(\alpha-\beta-2 \varepsilon_{4}\right) G_{\alpha, \beta} \varepsilon(t) d \alpha d \beta \\
& +\frac{E_{D}}{2} \iint_{\alpha \geq \beta} \delta\left(\alpha-\beta-2 \varepsilon_{4}\right) H(\alpha+\beta) G_{\alpha, \beta} \varepsilon(t) d \alpha d \beta .
\end{aligned}
$$

Geometric interpretation of Eq. (6) is given in the form of the Preisach triangle, which represents a part of halfplane $\alpha-\beta$ (Fig. 5). The hypotenuse of right Preisach triangle is a part of the line $\alpha=\beta$ and the vertex of its right angle has coordinates $\alpha=\varepsilon_{D}$ and $\beta=-\varepsilon_{D}$. The Preisach function is only supported along parallel lines $\alpha-\beta=0, \alpha-\beta-2_{\varepsilon 1}=0$, $\alpha-\beta-2_{\varepsilon 2}=0, \alpha-\beta 2_{\varepsilon 3}=0, \alpha-\beta 2_{\varepsilon 4}=0$, and $\alpha-\beta 2_{\varepsilon 5}=0$, and is zero in every other point of limiting triangle.

The elimination of the parameter $\beta$ from the Eq. (6) and the transition to a single integral is possible, since in the first part of that expression $\beta=\alpha$, in the second $\beta=\alpha-2_{\varepsilon 1}$,

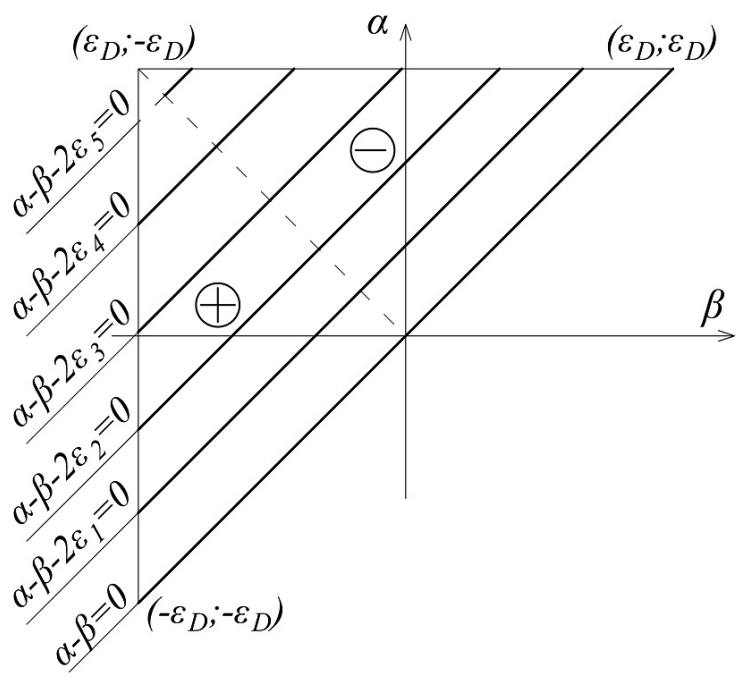

Fig. 5 Single crystal Preisach triangle

in the third $\beta=\alpha-2_{\varepsilon 2}$, in the fourth $\beta=\alpha-2_{\varepsilon 3}$, in the fifth $\beta=\alpha-2_{\varepsilon 4}$, and in the last $\beta=\alpha-2_{\varepsilon 5}$, which gives the Eq. (7).

$\sigma(t)=\frac{E}{2} \int_{-\varepsilon_{D}}^{\varepsilon_{D}} G_{\alpha, \alpha} \varepsilon(t) d \alpha-\frac{E}{2} \int_{2 \varepsilon_{1}-\varepsilon_{D}}^{\varepsilon_{D}} G_{\alpha, \alpha-2 \varepsilon_{1}} \varepsilon(t) d \alpha$

$+\frac{E_{a}}{2} \int_{2 \varepsilon_{2}-\varepsilon_{D}}^{\varepsilon_{D}} G_{\alpha, \alpha-2 \varepsilon_{2}} \varepsilon(t) d \alpha+\frac{E_{h}-E_{a}}{2} \int_{2 \varepsilon_{3}-\varepsilon_{D}}^{\varepsilon_{D}} G_{\alpha, \alpha-2 \varepsilon_{3}} \varepsilon(t) d \alpha$

$\frac{E_{h}}{2} \int_{2 \varepsilon_{4}-\varepsilon_{D}}^{\varepsilon_{D}} G_{\alpha, \alpha-2 \varepsilon_{4}} \varepsilon(t) d \alpha+\frac{E_{D}}{2} \int_{2 \varepsilon_{5}-\varepsilon_{D}}^{\varepsilon_{D}} G_{\alpha, \alpha-2 \varepsilon_{5}} \varepsilon(t) d \alpha$,

where last part of Eq. (7) exists only for $\alpha \geq 0$.

3 Polycrystalline Preisach model and monotonic axial response of structural mild steel with damage

The existence of crystals with different properties is a condition for the material anisotropy. Adopting that material crystals are oriented in the same direction, it is possible to speak of the quasi-isotropy of material.

Parallel connection of infinitely many mechanical models, shown in Fig. 6, provides the polycrystalline material model according to Iwan [16], where a parallel connection is the consequence of a strain as an input. Adopting unequal material characteristics for each element, real material behavior was obtained.

For a system of infinitely many parallel-connected units, with different yield limits $Y_{i}^{\min } \leq Y_{i} \leq Y_{i}^{\max }$ and different characteristics of delay elements $\varepsilon_{d}{ }^{\text {init }} \leq \varepsilon_{d} \leq \varepsilon_{d}^{\text {full }}$ expression for the total stress is:

$$
\sigma(t)=\Sigma \int_{Y_{i}^{\min }}^{Y_{i}^{\max }} p\left(Y_{i}\right) \sigma\left(Y_{i}, t\right) d Y_{i}+\int_{\varepsilon_{d}^{\text {init }}}^{\varepsilon_{d}^{\text {full }}} p\left(\varepsilon_{d}\right) \sigma\left(\varepsilon_{d}, t\right) d \varepsilon_{d},
$$


where $\sigma\left(Y_{i}, t\right)$ is stress corresponding to the individual unit of the yield limit $Y_{i}, p\left(Y_{i}\right)$ is the distribution function of the yield limit, $\sigma\left(\varepsilon_{d}, t\right)$ is stress corresponding to the unit with strain delay $\varepsilon_{d}$, while $p\left(\varepsilon_{d}\right)$ is its distribution function.

The material model, composed of units with the same Young's modulus $E$, the length of yield plateau $\varepsilon_{L}$, and the hardening modulus $E_{a}$ and $E_{h}$, but different yield limits $Y_{i}$, is defined. The accurate description of the monotonic response of the structural mild steel under axial load is granted by assuming that the yield limits $Y_{1}=Y_{2}$ are same in all parallel-connected individual units.

Defining that the distribution functions of other $Y_{i}$ values are uniform, as in papers [12, 13], and [17]:

$$
p\left(Y_{i}\right)=\frac{1}{Y_{i}^{\max }-Y_{i}^{\min }}=\text { const },
$$

as well distribution function of delay strain $\varepsilon_{d}$ :

$$
p\left(\varepsilon_{d}\right)=\frac{1}{\varepsilon_{d}^{\text {full }}-\varepsilon_{d}^{\text {init }}}=\text { const },
$$

the total stress, due to strain as an input, becomes:

$$
\begin{aligned}
& \sigma(t)=\frac{E}{2}\left[\int_{-\varepsilon_{u}}^{\varepsilon_{D}} G_{\alpha, \alpha} \varepsilon(t) d \alpha-\int_{2 \varepsilon_{1}-\varepsilon_{D}}^{\varepsilon_{D}} G_{\alpha, \alpha-2 \varepsilon_{1}} \varepsilon(t) d \alpha d Y_{1}\right. \\
& +\frac{E_{a}}{E} \int_{2 \varepsilon_{2}-\varepsilon_{D}}^{\varepsilon_{D}} G_{\alpha, \alpha-2 \varepsilon_{2}} \varepsilon(t) d \alpha \\
& +\frac{E_{h}-E_{a}}{E} p\left(Y_{3}\right) \int_{Y_{3}^{\min }}^{Y_{3}^{\max }} \int_{2 \varepsilon_{3}-\varepsilon_{D}}^{\varepsilon_{D}} G_{\alpha, \alpha-2 \varepsilon_{3}} \varepsilon(t) d \alpha d Y_{3} \\
& -\frac{E_{h}}{E} p\left(Y_{4}\right) \int_{Y_{3}^{\text {min }}}^{Y_{3}^{\text {max }}} \int_{\varepsilon_{4}-\varepsilon_{D}}^{\varepsilon_{D}} G_{\alpha, \alpha-2 \varepsilon_{4}} \varepsilon(t) d \alpha d Y_{4} \\
& +\frac{E_{D}}{E} p\left(\varepsilon_{d}\right) \int_{\varepsilon_{d}^{\text {mit }}}^{\varepsilon_{d \varepsilon_{5}}^{\text {fill }}} \int_{2 \varepsilon_{5}-\varepsilon_{D}}^{\varepsilon_{D}} G_{\alpha, \alpha-2 \varepsilon_{5}} \varepsilon(t) d \alpha d \varepsilon_{d} .
\end{aligned}
$$

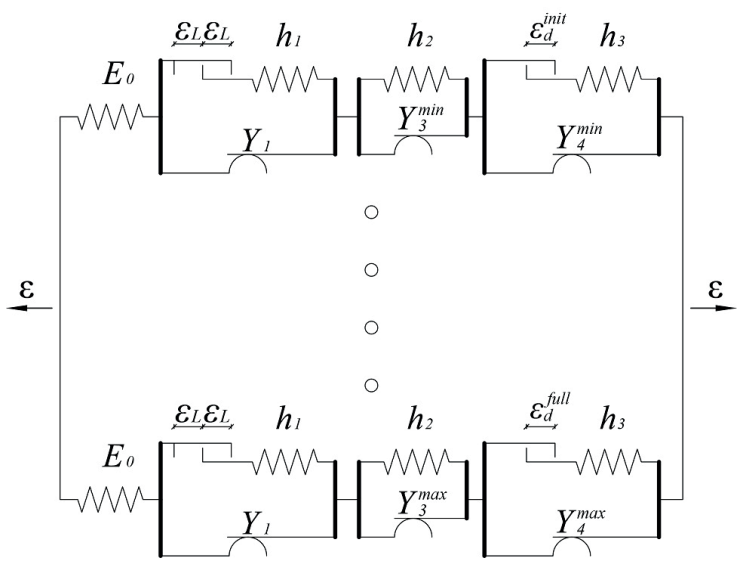

Fig. 6 Parallel connection of infinitely many unit models with different yield limits $Y_{i}^{\min } \leq Y_{i} \leq Y_{i}^{\max }$
The first addend of the Eq. (11) does not depend on $Y_{i}$ and based on other addends, following equalities hold respectively: $\alpha-\beta=2_{\varepsilon 1}, \alpha-\beta=2_{\varepsilon 2}, \alpha-\beta=2_{\varepsilon 3}, \alpha-\beta=2_{\varepsilon 4}$, and $\alpha-\beta=2_{\varepsilon 5}$.

Finally, parameter $\beta$ can be introduced into the expression again, with the changes $-d \beta \cdot\left(E_{a} / 2\right)=d Y_{3}$ and $-d \beta \cdot\left(E_{h} / 2\right)=d Y_{4}$, where the negative sign of the change is lost to the shift of the integration boundaries:

$$
\begin{aligned}
& \sigma(t)=\frac{E}{2}\left[\int_{-\varepsilon_{u}}^{\varepsilon_{u}} G_{\alpha, \alpha} \varepsilon(t) d \alpha-\int_{2 \varepsilon_{1}-\varepsilon_{u}}^{\varepsilon_{u}} G_{\alpha, \alpha-2 \varepsilon_{1}} \varepsilon(t) d \alpha d Y_{1}\right] \\
& +\frac{E_{a}}{2} \int_{2 \varepsilon_{2}-\varepsilon_{D}}^{\varepsilon_{D}} G_{\alpha, \alpha-2 \varepsilon_{2}} \varepsilon(t) d \alpha \\
& +\frac{E_{a}\left(E_{h}-E_{a}\right)}{4} p\left(Y_{3}\right) \iint_{A} G_{\alpha, \beta} \varepsilon(t) d \alpha d \beta \\
& -\frac{E_{h}^{2}}{4} p\left(Y_{4}\right) \iint_{B} G_{\alpha, \beta} \varepsilon(t) d \alpha d \beta \\
& +\frac{E_{D}}{4} p\left(\varepsilon_{d}\right) \iint_{C} G_{\alpha, \beta} \varepsilon(t) d \alpha d \beta .
\end{aligned}
$$

The first part of the Eq. (12) is the elastic stress, which can be calculated as:

$$
\frac{E}{2} \int_{-\varepsilon_{d}}^{\varepsilon_{d}} G_{\alpha, \alpha} \varepsilon(t) d \alpha=\frac{E}{2}(\varepsilon-(-\varepsilon))=E \varepsilon
$$

The integration domains in the Eq. (12) represent the surface of the band between the corresponding lines in the restricted triangle. Domain A represents the surface between the lines $\alpha-\beta=2 \varepsilon_{3}{ }^{\text {init }}$ and $\alpha-\beta=2 \varepsilon_{3}$ full, while domain $\mathrm{B}$ represents the surface between the lines $\alpha-\beta=2 \varepsilon_{4}{ }^{\text {init }}$ and $\alpha-\beta=2 \varepsilon_{4}{ }^{\text {full }}$. Both domains represent the plastic region with nonlinear hardening. The softening region, the result of damage under tension is represented with domain $\mathrm{C}$. Integration domain $\mathrm{C}$ represents the surface of the region between lines $\alpha-\beta=2 \varepsilon_{5}^{\text {init }}, \alpha-\beta=2 \varepsilon_{5}^{\text {full }}$, and $\alpha+\beta=0$. Geometrical interpretation of Eq. (12) in the form of the Preisach triangle is given in Fig. 7.

Preisach function outside of domains A, B, and C, and lines $\alpha-\beta-=0$, and $\alpha-\beta-2 \varepsilon_{1}=0$ is equal to zero.

\section{Experimental results and model verification}

The experiments presented in the paper are conducted as part of structural mild steel cyclic behavior study. For the purpose of determining material characteristics, test coupons are subjected to axial monotonic tension up to failure at room temperature. The cylindrical specimens with dimensions shown in Fig. 8, are adopted according to [18]. To minimize surface roughness effects, finely polished surfaces have been used. 


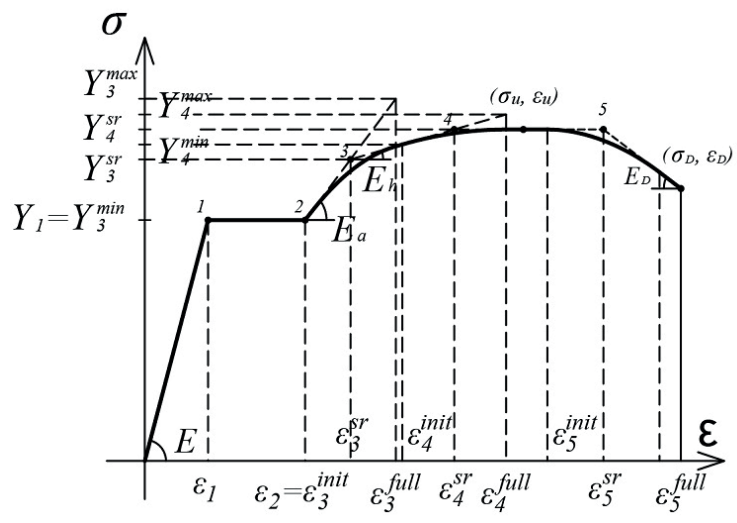

(a)

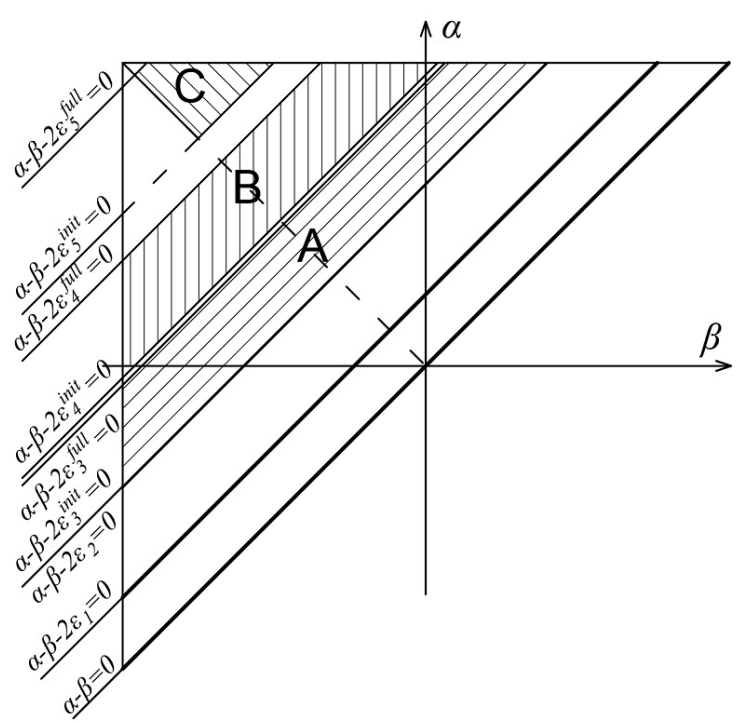

(b)

Fig. 7 a) The stress-strain curve of the material for the model defined by Eq. (12); b) The Preisach triangle for the material model defined by the Eq. (12)

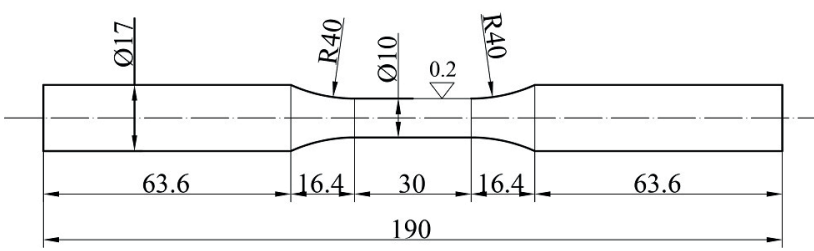

Fig. 8 Mechanical single crystal model

The following three steel grades have been considered for the study: S235, S275, and S355, all showing prominent yield plateau and characterized by Lüders band phenomenon. Two tests were performed for each steel grade, showing a suitable agreement. The test coupons meet European structural steel standard EN 10025: 2004 [19, 20].

The loading device is SHIMADZU ServoPulser which is universal tension and compression fatigue testing machine, providing stocky configuration, fine alignment, and restraint of lateral movement of cross-heads. A strain is measured by tension extensometer SHIMADZU SG 50-100, whose gauge length is $25 \mathrm{~mm}$ (Fig. 9). All tests are conducted with constant displacement velocity of $2 \mathrm{~mm} / \mathrm{min}$.

Experimental tests conducted under tension up to failure displayed similar behavior of the test specimens of the same steel grade, therefore just one test of the same steel grade is presented in Fig. 10.

Formation of the Lüders band phenomenon is noticeable for all three types of steel, as so softening region due to damage under tension. The test shows the growth of yield limit, ultimate stress, and fracture stress with the increase of steel grade. Yield strengths meet the requirements for engineering application. Material characteristics of all three steel grades obtained from tests and used for Preisach model formation were given in Table 1.

The proposed model Eq. (12) with parameters in Table 1 is validated against experimental results, through data comparison given in Fig. 11 for all three types of structural mild steel with prominent yield plateau.

\section{Conclusions}

This model represents simplified and easy to use a new type of Preisach model that characterize structural steel response under axial monotonic load. The present paper

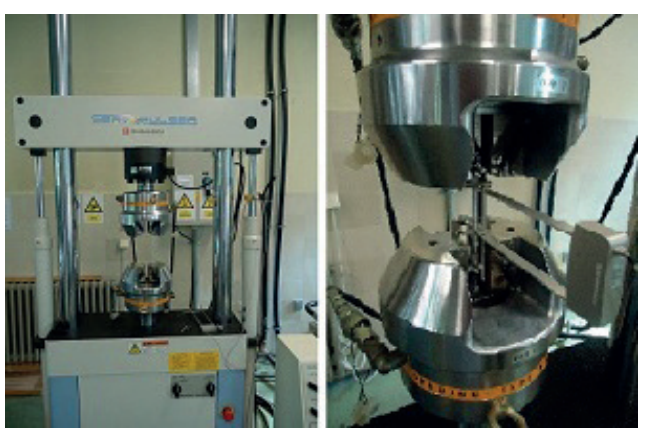

Fig. 9 Test equipment configuration

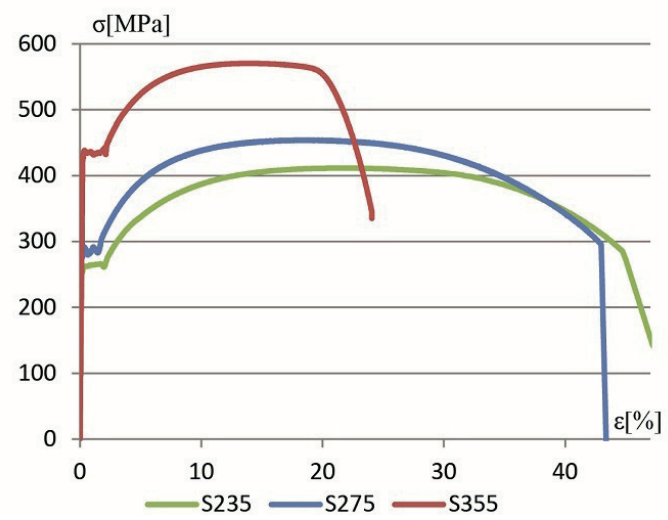

Fig. 10 Experimental monotonic loading curves of S235, S275, and S355 steel grade coupons 
Table 1 - Main mechanical properties of specimens

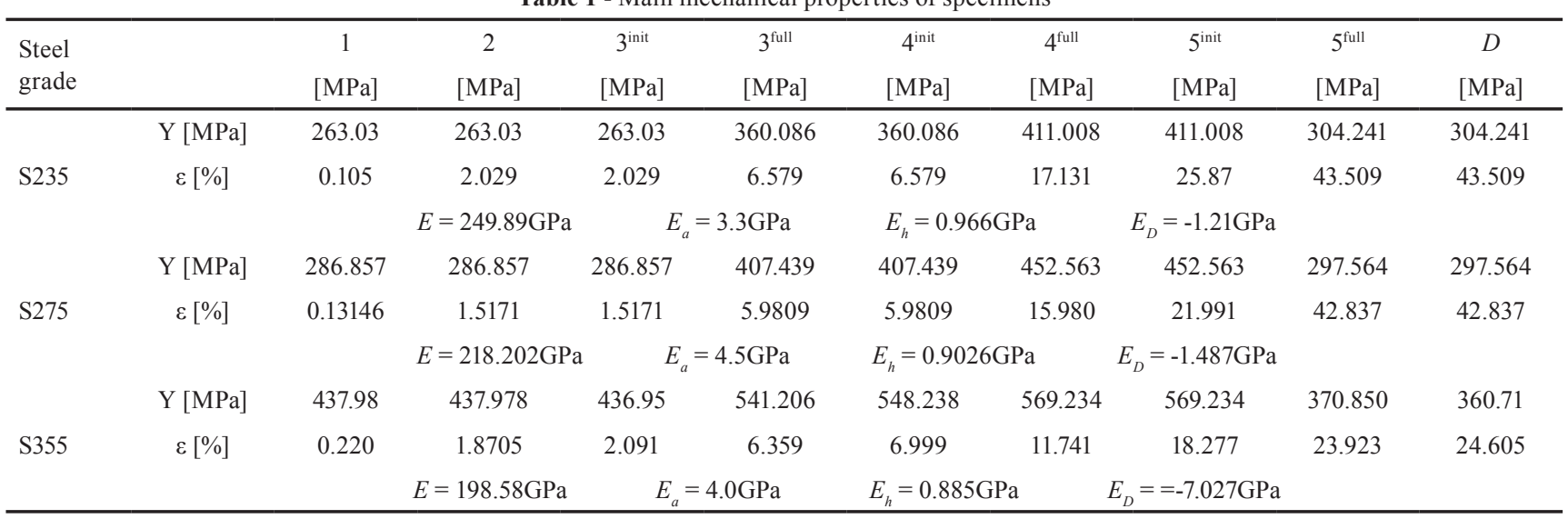
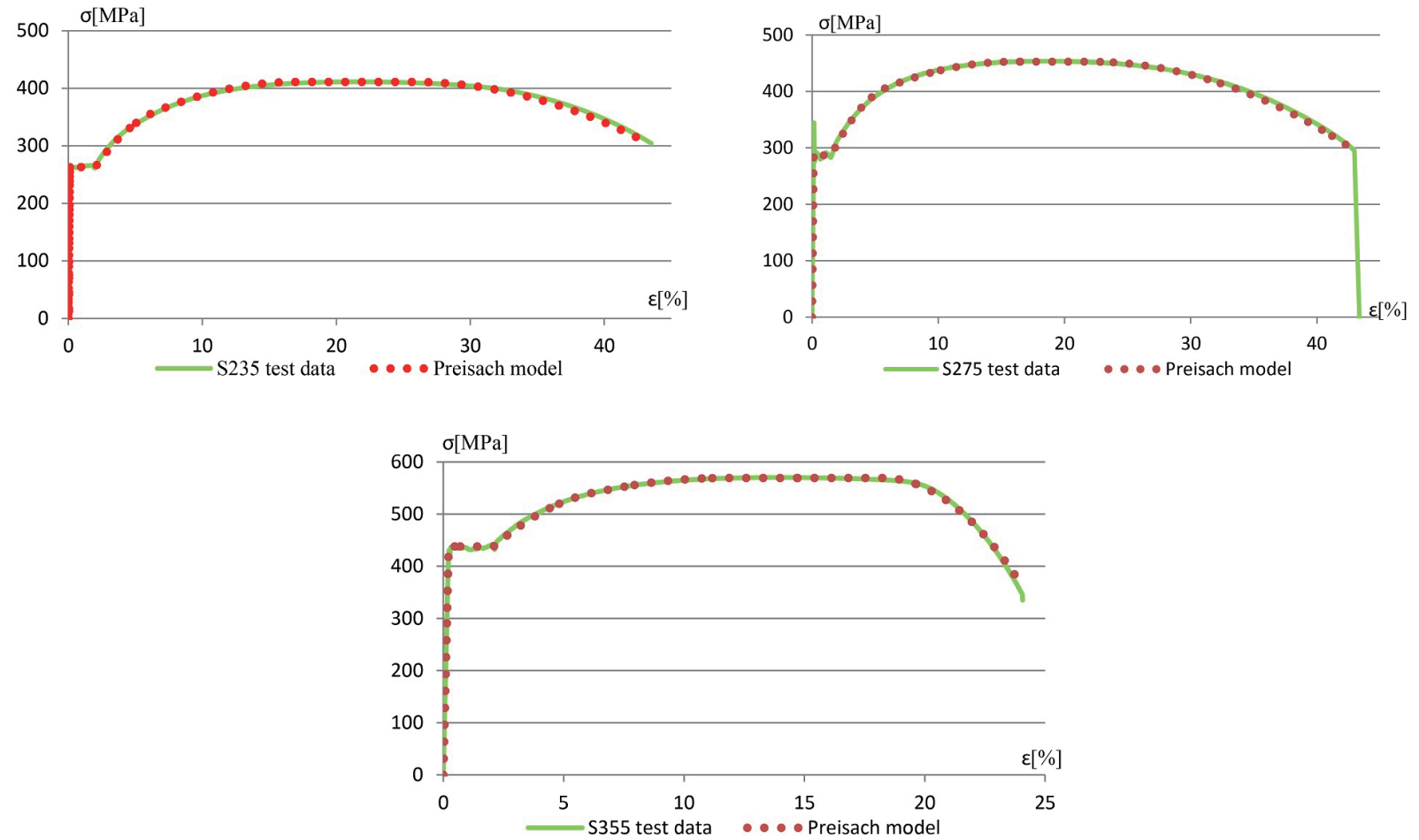

Fig. 11 Comparison of numerical and experimental results for all three types of steel grades

focuses on the application of the Preisach model to the response of the structural mild steel with prominent yield plateau under monotonic load accounting damage occurrence under tension only. The main advantage of the proposed model is a simple and rigorous mathematical formulation that provides pure geometrical interpretation, easy for use, in the form of the Preisach triangle. This model provides innovative and simplified formulation of the softening region including spring element with negative stiffness in the mechanical model. The first delay element in the mechanical model provides the formation of yield plateau characterized for Lüders band phenomenon, while with the second element different material behavior under tension and compression is provided.
Because of the inherent features of Preisah type models, this model possesses memory, congruency, and wiping out properties. Further extension of the model is needed in order to comprehend cyclic phenomena such a vanishing of yield plateau and cyclic hardening/softening.

The comparison made with experimental results showed that the numerical model can reproduce tension loading paths with very good accuracy for mostly used European structural steel grades S235, S275, and S355.

\section{Acknowledgment}

Authors are grateful for the financial support of the Ministry of education, science and technological development of the Republic of Serbia within the project III 42012. 


\section{References}

[1] Srinivasan, N., Raghu, N., Venkatraman, B. "Study On The Deformation Band Characteristics In Mild Steel Using Digital Image Correlation", Journal of Multidisciplinary Engineering Science and Technology (JMEST), 1(3), pp. 400-403, 2014. [online] Available at: https://www.jmest.org/wp-content/uploads/JMESTN42350306. pdf [Accessed: 20 January 2020]

[2] Hertzberg, R. W. "Deformation and Fracture Mechanics of Engineering Materials", 4th ed., John Wiley \& Sons, Hoboken, NJ, USA, 1996.

[3] Zhang, Y. T., Ao, T., Jiao, W., Cui, Y. H. "Prediction of the Lüders band in fine grained steel strips under uniaxial tension", Computational Materials Science, 41(4), pp. 547-552, 2008. https://doi.org/10.1016/j.commatsci.2007.05.011

[4] Yoshida, F. "A constitutive model of cyclic plasticity", International Journal of Plasticity, 16(3-4), pp. 359-380, 2000. https://doi.org/10.1016/S0749-6419(99)00058-3

[5] Carrella, A., Brennan, M. J., Waters, T. P. "Static analysis of a passive vibration isolator with quasi-zero-stiffness characteristic", Journal of Sound and Vibration, 301(3-5), pp. 678-689, 2007. https://doi.org/10.1016/j.jsv.2006.10.011

[6] Danh, L. T., Ahn, K. K. "Active pneumatic vibration isolation system using negative stiffness structures for a vehicle seat", Journal of Sound and Vibration, 333(5), pp. 1245-1268, 2014. https://doi.org/10.1016/j.jsv.2013.10.027

[7] Platus, D. L. "Negative-stiffness-mechanism vibration isolation systems", In: Proceedings of the SPIE 1619, Vibration Control in Microelectronics, Optics and Metrology, San Jose, CA, USA, 1992, pp. 44-54.

https://doi.org/10.1117/12.56823

[8] Carrella, A., Brennan, M. J., Waters, T. P., Shin, K. "On the design of a high-static-low-dynamic stiffness isolator using linear mechanical springs and magnets", Journal of Sound and Vibration, 315(3), pp. 712-720, 2008

https://doi.org/10.1016/j.jsv.2008.01.046

[9] Zhou, N., Liu, K. "A tunable high-static-low-dynamic stiffness vibration isolator", Journal of Sound and Vibration, 329(9), pp. 1254-1273, 2010. https://doi.org/10.1016/j.jsv.2009.11.001
[10] Lakes, R. S. "Extreme Damping in Composite Materials with a Negative Stiffness Phase", Physical Review Letters, 86(13), pp. 2897-2900, 2001. https://oi.org/10.1103/PhysRevLett.86.2897

[11] Preisach, F. "About the magnetic aftereffect (Über die magnetische Nachwirkung)", Zeitschrift für Physik, 94, pp. 277-302, 1935. (in German) https://doi.org/10.1007/BF01349418

[12] Lubarda, A. V., Sumarac, D., Krajcinovic, D. "Hysteretic Response of Ductile Materials Subjected to Cyclic Loads", In: Ju, J. W. (ed.) Recent Advances in Damage Mechanics and Plasticity, 123, 1992, pp. 145-157.

[13] Lubarda, A. V., Sumarac, D., Krajcinovic, D. "Preisach Model and Hysteretic Behavior of Ductile Materials", European Journal of Mechanics- A/Solids, 12(4), pp. 445-470, 1993.

[14] Mayergoyz, I. D. "Mathematical Models of Hysteresis", Springer, New York, NY, USA, 1991. https://doi.org/10.1007/978-1-4612-3028-1

[15] Šumarac, D., Perović, Z. "Cyclic plasticity of trusses", Archive of Applied Mechanics, 85, pp. 1513-1526, 2015. https://doi.org/10.1007/s00419-014-0954-7

[16] Iwan, W. D. "On a Class of Models for the Yielding Behavior of Continuous and Composite Systems", Journal of Applied Mechanics, 34(3), pp. 612-617, 1967. https://doi.org/10.1115/1.3607751

[17] Sumarac, D., Stosic, S. "The Preisach model for the cyclic bending of elasto-plastic beams", European Journal of Mechanics - A/Solids, 15(1), pp. 155-172, 1996.

[18] ASTM "ASTM E606/E606M-12 Test Method for Strain-Controlled Fatigue Testing", ASTM International, West Conshohocken, PA, USA, 2012. https://doi.org/10.1520/E0606_E0606M-12

[19] CEN "EN10025-1 Hot Rolled products of structural steels - Part 1: Technical delivery conditions for non-alloy structural steels", European Committee for Standardization, Brussels, Belgium, 2004.

[20] CEN "EN10025-2 Hot Rolled products of structural steels - Part 2: General technical delivery conditions", European Committee for Standardization, Brussels, Belgium, 2004. 